\title{
Next-Generation Sequencing Reveals Downregulation of the Wnt Signaling Pathway in Human Dysmature Cumulus Cells as a Hallmark for Evaluating Oocyte Quality
}

\author{
Ryosuke Akino ${ }^{1,2,3,+}{ }^{\text {, Daisuke Matsui }}{ }^{1,4,+}$, Ryouka Kawahara-Miki ${ }^{5,+}$, Mitsuyoshi Amita ${ }^{1}$, \\ Kuniko Tatsumi ${ }^{1}$, Eri Ishida ${ }^{1}$, Woojin Kang ${ }^{1} \mathbb{D}$, Shuji Takada ${ }^{3}$, Kenji Miyado ${ }^{6}{ }^{\mathbb{D}}$, \\ Akihiko Sekizawa ${ }^{2} \mathbb{D}$, Takakazu Saito ${ }^{1}$, Tomohiro Kono ${ }^{7}$ and Hidekazu Saito ${ }^{1, *}$ \\ 1 Division of Reproductive Medicine, National Center for Child Health and Development, 2-10-1 Okura, \\ Setagaya, Tokyo 157-8535, Japan; akkino1103@med.showa-u.ac.jp (R.A.); damatsui_20@yahoo.co.jp (D.M.); \\ amita-m@ncchd.go.jp (M.A.); tatsumi-k@ncchd.go.jp (K.T.); ishida-e@ncchd.go.jp (E.I.); \\ kwjbear@gmail.com (W.K.); saito-tk@ncchd.go.jp (T.S.) \\ 2 Department of Obstetrics and Gynecology, Showa University School of Medicine, 1-5-8 Hatanodai, \\ Shinagawa, Tokyo 142-8666, Japan; sekizawa@med.showa-u.ac.jp \\ 3 Department of Systems BioMedicine, National Research Institute for Child Health and Development, \\ 2-10-1 Okura, Setagaya, Tokyo 157-8535, Japan; takada-s@ncchd.go.jp \\ 4 Rose Ladies Clinic, 2-3-18 Todoroki, Setagaya, Tokyo 158-0082, Japan \\ 5 Nodai Genome Research Center, Tokyo University of Agriculture, 1-1-1 Sakuragaoka, Setagaya, \\ Tokyo 156-8502, Japan; r3miki@nodai.ac.jp \\ 6 Department of Reproductive Biology, National Research Institute for Child Health and Development, \\ 2-10-1 Okura, Setagaya, Tokyo 157-8535, Japan; miyado-k@ncchd.go.jp \\ 7 Department of Bioscience, Tokyo University of Agriculture, 1-1-1 Sakuragaoka, Setagaya, \\ Tokyo 156-8502, Japan; tomohiro@nodai.ac.jp \\ * Correspondence: saitou-hi@ncchd.go.jp; Tel.: +81-3-5494-7047; Fax: +81-3-5494-7048 \\ + Authors contributed equally.
}

Received: 14 November 2020; Accepted: 26 November 2020; Published: 1 December 2020

\begin{abstract}
Background: Dysmature cumulus cells are lower fertilization rates and abnormalities in embryonic development compared to maturation cumulus cells. Morphological evaluation of cumulus-oocyte complexes (COCs) considered the possibility that differences may also be found in gene expression. Purpose: To identify hallmarks for evaluating oocyte quality by investigating gene expression patterns in human cumulus cells surrounding oocytes. Methods: Cumulus cells were obtained from the cumulus-oocyte complex of infertile women treated with assisted reproductive technology. Based on maturity level, the cumulus cells were classified into two categories, i.e., dysmature cumulus cell (DCC) and maturation cumulus cell. DCCs were subjected to gene expression analysis using next-generation sequencing and compared with COCs that are in the process of maturation as controls. Results: The expression levels of genes involved in the Wnt signal/ $\beta$-catenin pathway were significantly reduced in DCCs compared with those in control cells. Moreover, the expression levels of genes involved in multiple pathways associated with apoptosis were also significantly reduced compared with those in control cells. Conclusions: DCCs showed significant decreases in apoptosis- and Wnt/ $\beta$-catenin signaling-associated gene expression. DCCs could be classified by morphological evaluation, and the method described in this study may be useful as an oocyte quality estimation tool.
\end{abstract}

Keywords: cumulus cells; female infertility; next-generation sequencing; oocyte quality; Wnt signaling pathway 


\section{Introduction}

A human oocyte and its supportive cells, known as granulosa cells, are interdependent throughout ovarian development and ovulation for successful fertilization [1]. Oocytes isolated from primordial follicles fail to grow without the aid of granulosa cells [2,3]. Cumulus cells are the innermost subgroup of granulosa cells surrounding the oocyte and play a key role in oocyte maturation and fertilization. Cumulus cell function depends on the gap junctions between cumulus cells and the oocyte, which allow rapid transfer of molecules to the oocyte [4,5]; so, the quality of cumulus cells is thought to reflect oocyte quality. Therefore, transcriptomic profiling of cumulus cells is useful in identifying biomarkers indicating the developmental ability of oocyte maturation (Figure 1a).
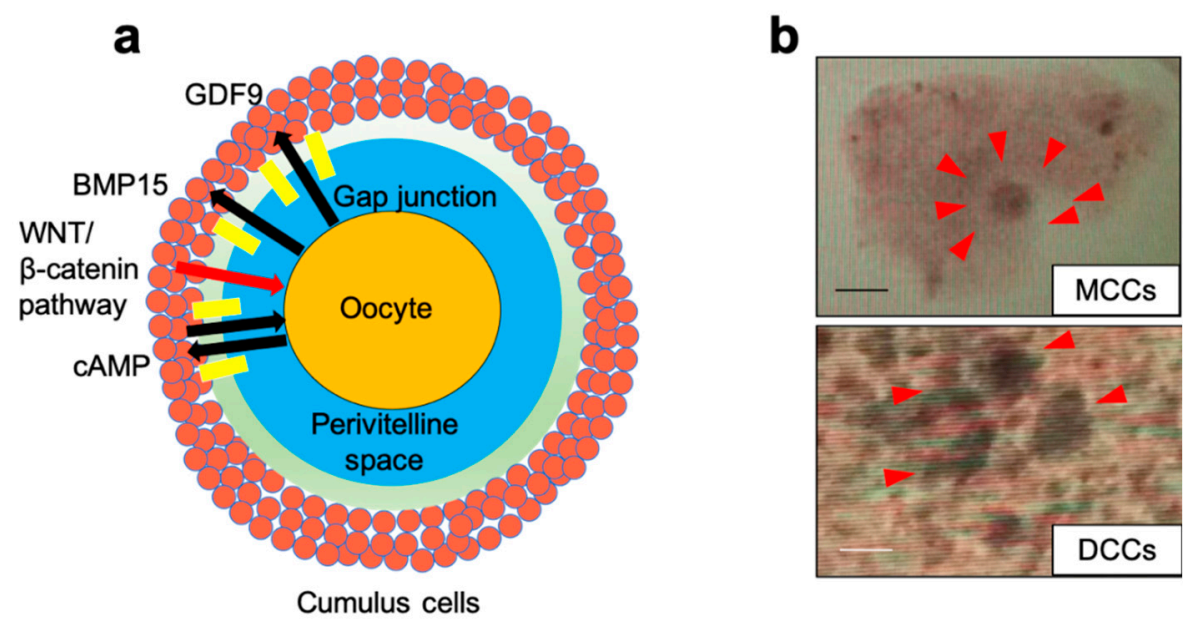

Figure 1. Close interaction between cumulus cells and the oocyte. (a) Schematic overview of cumulus cells surrounding an oocyte. Two-way communication between cumulus-oocyte complexes (COCs) and an oocyte shows important roles such as the transmission of genetic information and supply of raw materials to early embryos. Growth differentiation factor 9 (GDF9) and bone morphogenetic protein 15 (BMP15) from the superfamily of transforming growth factor- $\beta$ (TGF- $\beta$ ) are expressed in an oocyte-specific mode from a very early stage and play an essential role in promoting follicle growth beyond the primary stage. Wnt/ $\beta$-catenin signaling pathway controls various developmental processes and is thought to play an important role in human folliculogenesis. (b) Classification of COCs. The maturation cumulus cell (MCC) is a complete expansion of cumulus with a visible halo. A dysmature cumulus cell (DCC) is an incomplete or complete expansion and dissociation of cumulus layers with dark spots. Scale bars $=100 \mu \mathrm{m}$.

The classification of mature cumulus-oocyte complexes (COCs), based on morphology, was first described in the 1980s [6] and has been used for many decades [7-10].

In the human menstrual cycle, only less than $1 \%$ of follicles lead to ovulation, and more than $99 \%$ of follicles lead to atretic follicles [11]. We have used these criteria for more than a decade and reported that the oocytes classified as "dysmature" exhibit a remarkably lower fertilization rate, compared to COCs that are in the process of maturation [12]. Our previous studies revealed significant differences in gene expression of DCCs compared to COCs that are in the process of maturation [13], and DCCs are reportedly associated with apoptosis [13] and autophagy-independent cell death [1,12].

To date, several studies using next-generation sequencing (NGS) to examine cumulus cells or granulosa cells have been conducted. Using NGS, it is possible to comprehensively investigate gene expression in samples, because it can determine millions of sequences at once. For example, Hu et al. [14] reported the cause of insulin resistance in polycystic ovarian syndrome (PCOS) analyzing cumulus cells. Xu et al. [15] searched the difference in gene expression of mouse cumulus cells with and without blastocyst formation. Fuchs et al. [16] studied the difference in the gene expression of human cumulus granulosa cells based on the types of drugs used to trigger IVF cycles. Although other 
studies using NGS for cumulus cells are also present $[17,18]$, there have been a few studies on the gene expression related to COC maturity.

As described above, the evaluation of COC maturation is useful to estimate oocyte quality. Furthermore, the procedure used to assess cumulus cells is more straightforward than assessing the oocyte without damaging it. Nevertheless, there have been a few studies that estimate the difference in gene expression based on the maturity of COCs.

In the present study, we compared DCCs, which would become atretic follicles, with COCs that are in the process of maturation, and used NGS to search for candidate genes associated with follicular development, lower fertilization rate, apoptosis, and autophagy.

\section{Materials and Methods}

\subsection{Patients}

This study was reviewed and approved by the ethical committee of the National Center for Child Health and Development (\# 581, 3 July 2012). Women presenting unexplained infertility aged 33-42 years who underwent assisted reproductive technology (ART) were included. Briefly, cumulus cells were collected from patients who consented to participate in this study. Based on the morphological criteria, DCCs were selected, and COCs that are in the process of maturation were categorized as control (Supplemental Table S1). Twenty samples of cumulus cells were collected from patients who provided consent for their samples to be used for research purposes.

The patients underwent standard in vitro fertilization (IVF) treatment, which included gonadotropin administration to induce multiple follicular development, and transvaginal oocyte aspiration. COC retrieval was performed by vaginal puncture under ultrasound echo-guidance $36 \mathrm{~h}$ after human chorionic gonadotropin (hCG) administration (10,000 IU). The classification of COCs was based on the following morphological criteria: We defined DCCs as the presence of dark spots in the layers of cumulus cells, with or without complete expansion with a visible halo of the cumulus layer, to distinguish them from maturation cumulus cells (Figure 1b). The cumulus cells were mechanically removed at $40 \mathrm{~h}$ after hCG administration, before conventional IVF or intracytoplasmic sperm injection, washed in Sydney IVF fertilization (COOK Medical, Brisbane, Australia) medium, and frozen individually before total RNA extraction. The sample collection flow is shown in Figure 2.

\subsection{RNA Extraction and NGS}

Total RNA of cumulus cells from oocytes were extracted using the RNeasy mini kit (Qiagen, Valencia, CA, USA) following the manufacturer's protocol. The extracted RNA quality and quantity were assessed on a 2100 Bioanalyzer, using the RNA 6000 Nano kit (Agilent Technologies, Palo Alto, CA, USA). Libraries were prepared using a TruSeq RNA Sample Preparation Kit (Illumina Inc., San Diego, CA, USA) to generate clusters on the Illumina cBot (Illumina). Sequencing was performed on the Hiseq 2000/2500 (Illumina) as 50-bp reads, while image analysis and base calling were performed with CASAVA ver.1.7.0/1.8.2 (Illumina), following the manufacturer's instructions. High-quality sequences, which passed the default quality-filtering parameters in the Illumina pipeline GERALD stage were retained and exported into Strand NGS (Agilent Technologies) for subsequent data analysis. The next-generation Web sequence data were deposited in the Japanese Genotype-phenotype Archive (JGA) [Accession \#: JGAS00000000212].

Sequences were aligned with the human genome sequence (Feb. 2009/hg19), using the gene annotation file downloaded from the UCSC sequence and annotation database (http://hgdownload.cse. ucsc.edu/downloads.html\#human), and Strand NGS (Agilent Technologies). The downloaded UCSC annotation database included 40,437 genes and transcripts consisting of 19,805 protein-coding genes, 12,221 pseudogenes, and 7365 non-coding RNA (Supplemental Table S2). 


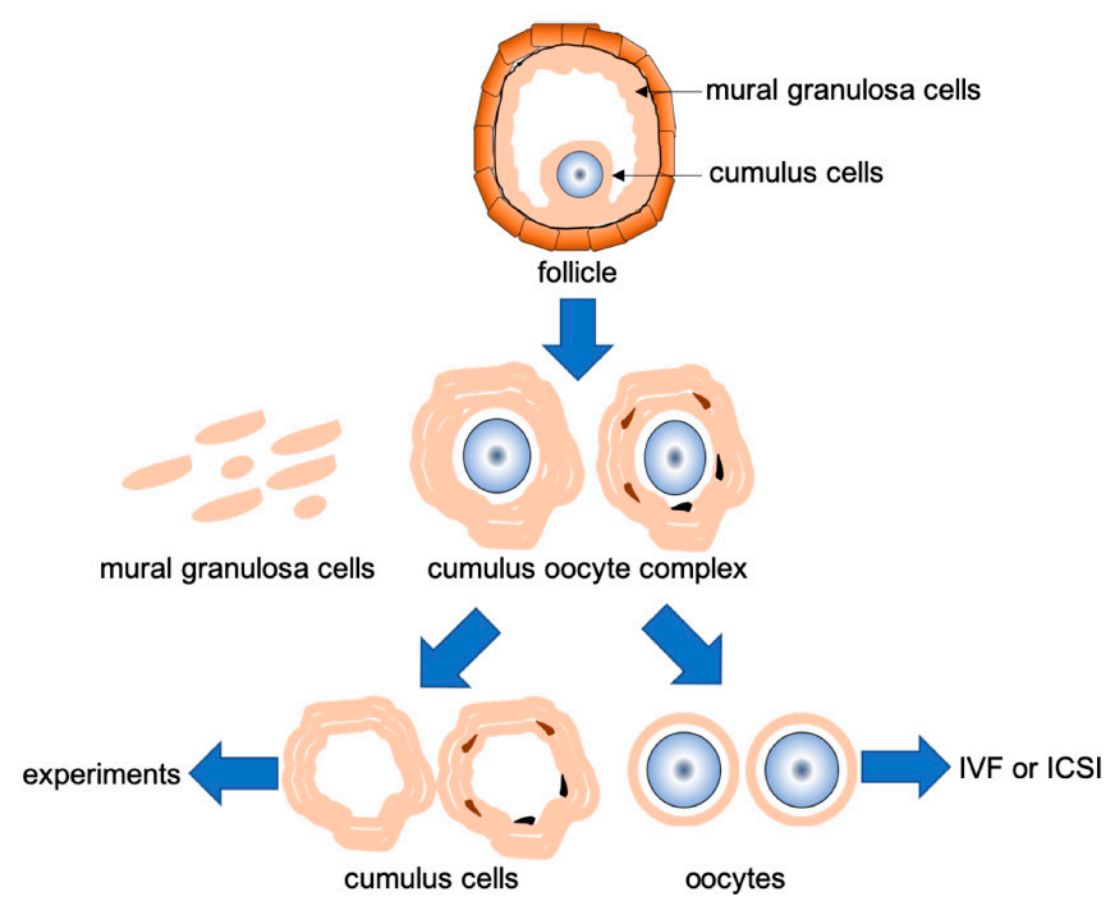

Figure 2. Flow chart for collecting samples. Flow chart of collecting cumulus cells from cumulus-oocyte complex (COC). COCs were retrieved from stimulated follicle by hormones. Mural granulosa cells and COCs were isolated from follicular aspirates. The maturity of COCs was evaluated based on morphologic criteria. Evaluated cumulus cells were physically detached from COCs. Obtained cumulus cells were used in experiments and oocytes were used for assisted reproductive technology (ART) by in vitro fertilization (IVF) or Intracytoplasmic sperm injection (ICSI).

\subsection{Gene Expression Profiling Analysis}

Uniquely aligned reads were quantified, and both gene and exon-level reads per kilobase per million mapped read (RPKM) values were calculated. The RPKM values represent counts of reads mapping a feature (gene, or exon), normalized to both overall sequencing coverage and the size of the feature [19].

Before gene expression analysis and the use of log-transformed and base-lined counts, we performed principal component analysis (PCA) and hierarchical clustering to overview the expression profiles of all samples. The values were normalized to mean 0 and variance 1 before applying log transformation.

Differentially expressed genes in DCCs were identified based on a 2-fold change compared to the mean value of the control, and statistical significance based on $p$-value $<0.05$, using one-way analysis of variance (ANOVA), followed by post hoc analysis using Student-Newman-Keuls. The list of differentially expressed genes was utilized for gene ontology (GO) analysis for biological/molecular function, using Strand NGS with a false discovery rate-corrected $p$-value $<0.01$. Furthermore, the list of differentially expressed genes was analyzed for pathway enrichment analysis using WikiPathway, and statistical significance was set at a $p$-value $<0.05$.

\subsection{Immunofluorescence}

Cumulus cells prepared as described above were permeabilized with $0.5 \%$ Triton X-100 in phosphate-buffered saline (PBS) for $5 \mathrm{~min}$ at $25^{\circ} \mathrm{C}$ and blocked with $1 \%$ BSA and $0.1 \%$ Tween 20 in PBS for $1 \mathrm{~h}$ at $25^{\circ} \mathrm{C}$. The samples were incubated overnight at $4{ }^{\circ} \mathrm{C}$ with monoclonal anti- $\beta$-Catenin-Cy3 conjugate (C7738, Sigma, St Louis, MO, USA), prepared at a 1:500 dilution in Dako antibody diluents (Dako, Carpinteria, CA, USA). The complexes were then washed three times with $0.1 \%$ Tween 20 in PBS. The nuclei were then counterstained with 4' ${ }^{\prime}, 6$-diamidino-2-phenylindole (DAPI) at a final 
concentration of $10 \mu \mathrm{g} / \mathrm{mL}$ in $0.1 \%$ Tween 20 in PBS for $30 \mathrm{~min}$ at $25^{\circ} \mathrm{C}$. Thereafter, samples were washed three times with $0.1 \%$ Tween 20 in PBS, immersed in Vectashield (Vector Laboratories Inc., Burlingame, CA, USA), and covered with coverslips. Microphotographs were captured using a confocal laser scanning microscope LSM510 Meta (Carl Zeiss MicroImaging, Inc., Thornwood, NY, USA). Photographs captured from three different areas of each sample were analyzed using Olympus DP Controller and DP Manager (Olympus, Tokyo, Japan). In each experiment, at least 300 cells were measured in randomly selected microscopic fields.

\section{Results}

\subsection{Sequencing and Mapping Summary}

Sequencing data for each sample resulted in approximately 10-27 M reads (Supplemental Table S3). Among the raw sequence reads, $77-85.5 \%$ aligned with the human genome sequence (Supplemental Table S3). Read counts were converted to RPKM [19] to assess global gene expression profiles. Based on the gene expression patterns, PCA was carried out, followed plotting 20 samples that were divided into DCCs and control. The results showed that samples collected from DCCs were differentially located compared to the control (Supplemental Figure S1a).

\subsection{Differentially Expressed Genes}

To determine the statistical difference in gene expression between DCCs and the control, we performed a one-way ANOVA followed by pair-wise comparisons. Among genes with a statistical significance, we selected those with two-fold differential expression. The Venn diagrams show genes with both two-fold differential expression and a statistical significance threshold, analyzed using a one-way ANOVA. Among these, 165 genes were upregulated, while 363 were downregulated (Supplemental Figure S1b, Supplemental Table S4).

\subsection{GO and Pathway Analysis}

We performed GO analysis to examine the enrichment of biological processes, molecular function, and cellular components and identify biological characteristic for differentially expressed gene list. Upregulated genes were enriched in four functions: single-organism processes, cellular processes, metabolic processes, and response to stimuli. Whereas in downregulated genes, only the plasma membrane portion was enriched in cellular components (Supplemental Table S5).

In upregulated genes, biological processes such as mitotic prometaphase, mitotic metaphase and anaphase, cell cycle checkpoints, kinesins, and mitotic G1-G1-S phases were affected significantly (all, $p<0.0001$ ) (Supplemental Table S6). In contrast, the Wnt signaling pathway and pluripotency, the integrated pancreatic cancer pathway, platelet homeostasis, arachidonic acid metabolism, and the netrin-1 signaling pathway were significantly affected in downregulated genes (all, $p<0.01$ ) (Table 1 ).

Among downregulated genes, we focused on those associated with Wnt signaling, since they were reportedly associated with the state of oocytes and cumulus cells [20,21]. When relative values of gene expressions were estimated, the expressions of five genes were significantly reduced in DCCs. Two cytokines (WNT9B and WNT10B) were significantly downregulated in DCCs (4.095 vs. 1.0, and 2.444 vs. 1.0, DCCs and the control, respectively; $p=0.0007$ and $p=0.0001)$. Frizzled-7 (FZD7), one of the Wnt receptors, was expressed in cumulus cells, and its expression was significantly reduced in DCCs (2.052 vs. 1.0; $p<0.0001)$. AXIN2 binds to various proteins constituting the Wnt signaling pathway, and its expression was also significantly reduced in DCCs (2.150 vs. $1.0 ; p<0.0001)$. Besides, the expression of Cyclin D1 (CCND1), a representative gene among target genes associated with cell proliferation and the cell cycle, was also reduced in DCCs (2.835 vs. 1.0; $p=0.0001$ ) (Figure 3a). As depicted in Figure $3 b$, we presumed that these reduced gene expressions impair the transduction of the Wnt signaling pathway in the DCCs. 
Table 1. Downregulated pathways in DCCs.

\begin{tabular}{cccc}
\hline Pathway & $p$-Value & Matched Entities & $\begin{array}{c}\text { Pathway Entities of } \\
\text { Experiment Type }\end{array}$ \\
\hline Hs_Wnt_Signaling_Pathway_and_Pluripotency_ & 0.0000586 & 5 & 100 \\
WP399_74897 & & 4 & 200 \\
Hs_Integrated_Pancreatic_Cancer_Pathway_ & 0.009160941 & 2 & 31 \\
WP2377_71228 & 0.005834554 & 3 & 27 \\
Hs_Platelet_homeostasis_WP1885_77031 & 0.000182 & 3 & 42 \\
Hs_Arachidonic_acid_metabolism_ \\
WP2650_76814
\end{tabular}

Nine DCC samples were analyzed, and downregulated pathways were presented.

a

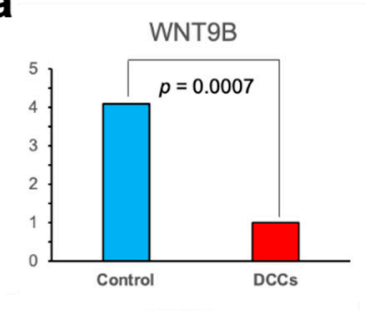

FZD7

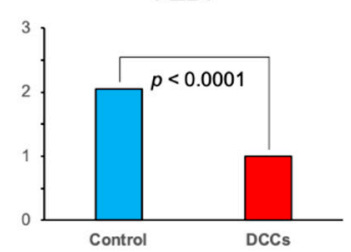

CCND1

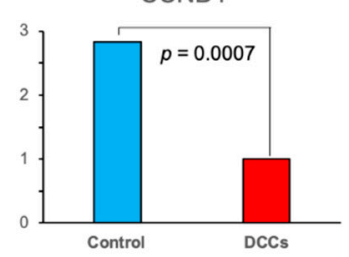

b
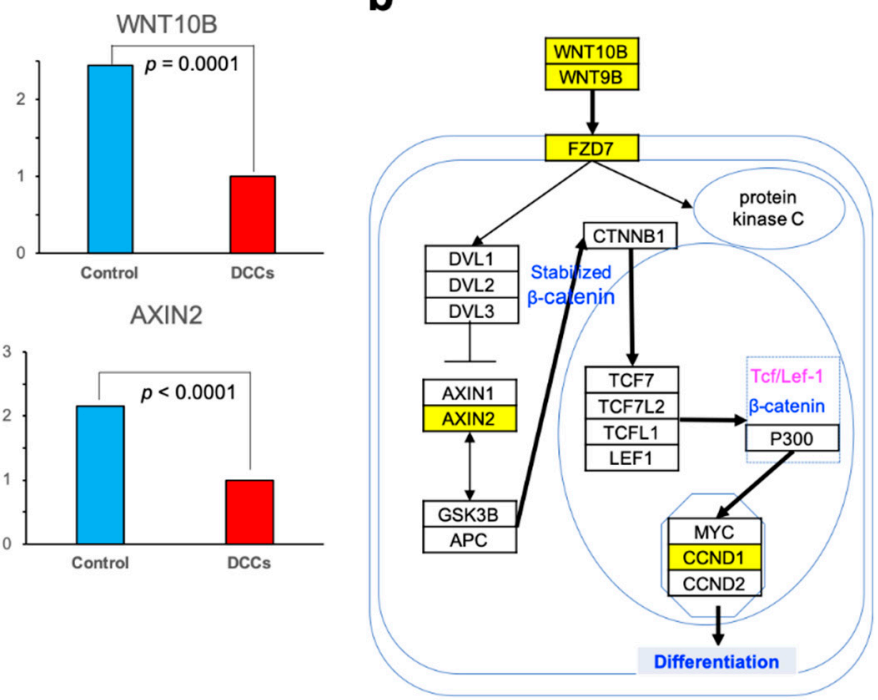

Figure 3. Wnt signaling-related genes downregulated in dysmature cumulus cells (DCCs). (a) Box plots showing the difference in gene expression in DCCs. The expression levels in DCCs were arbitrarily set at 1 . The graph indicates relative signal intensity in DCCs against that in control. The control is shown in blue and DCCs in red. (b) The Wnt signaling pathway. In this analysis, only five genes, denoted by yellow square, were statistically significant.

Based on the above results, we considered the possibility that the Wnt signaling pathway might not be acting only in DCCs compared to the control. Therefore, immunostaining was performed using $\beta$-catenin in DCC s and control. The cells were immunostained with $\beta$-catenin, and the nuclei were stained with DAPI. Of these cells, DCCs and control exhibited remarkable immunoreaction with $\beta$-catenin, but a difference in fluorescence patterns between these cells was also noted (Figure 4a).

Although $\beta$-catenin was diffusely localized in the cytoplasm of DCCs and control, it also appeared to be localized in intracellular structures, such as the nuclear membrane in DCCs (Figure 4b,c). The number of $\beta$-catenin-expressing cells was higher in DCCs than in control (Figure 4d). Our results suggested that excess $\beta$-catenin expression was similar in DCCs and control, but subcellular $\beta$-catenin localization was distinct between these cells, implying that the progression in CC maturation may 
result from altered localization $\beta$-catenin. Therefore, we assumed that these cells might be classified differently in different situations.
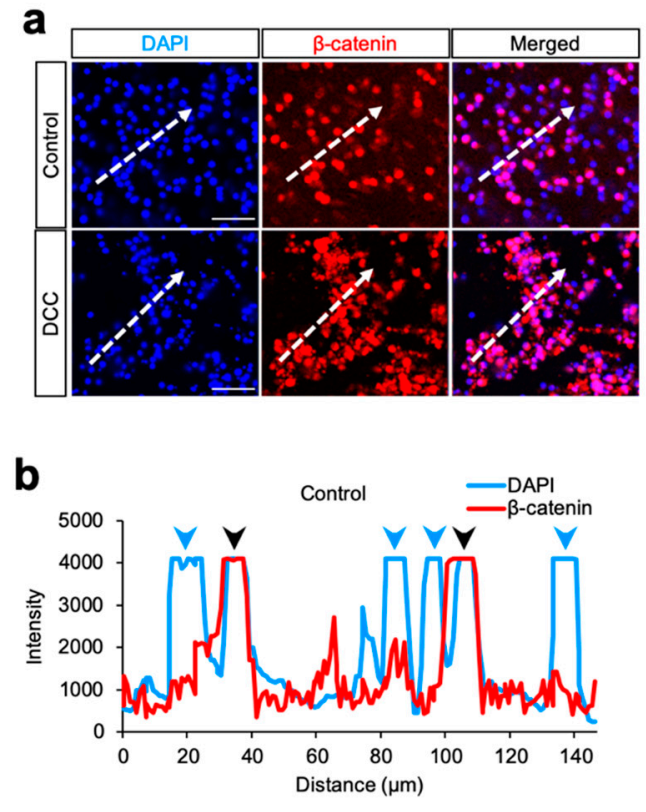

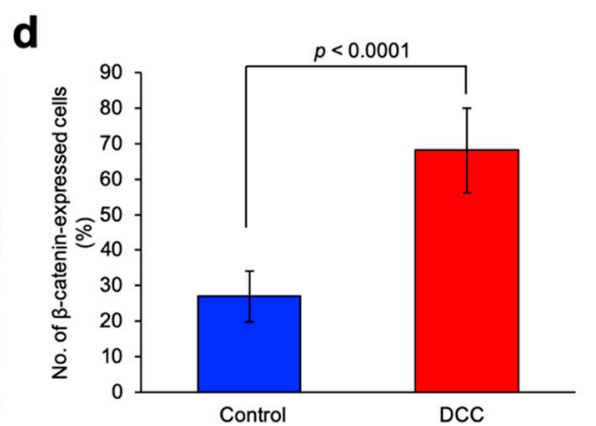

C

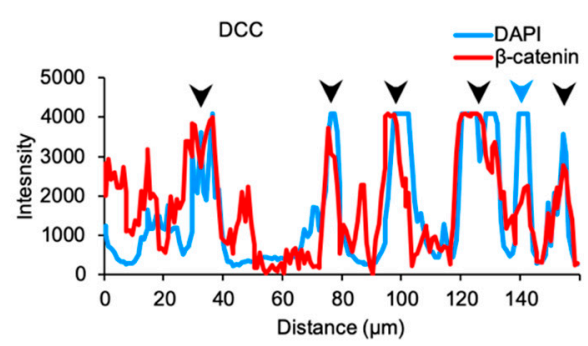

Figure 4. Expression of $\beta$-catenin. (a) Immunostaining of $\beta$-catenin; TUNEL assay was performed, and fluorescent images were captured. The red signal represents $\beta$-catenin. Blue signals represent $4^{\prime}$,6-diamidino-2-phenylindole (DAPI)-stained areas. Scale bars $=20 \mu \mathrm{m}$. (b) The fluorescence intensity profiles of $\beta$-catenin- and DAPI-stained areas in control. Fluorescence intensities were measured using white dotted lines. Red and blue lines represent $\beta$-catenin and DAPI, respectively. Three different areas of each sample were analyzed; the representative results are shown. (c) The fluorescence intensity profiles of $\beta$-catenin- and DAPI-stained areas in DCCs. Fluorescence intensities were measured using white dotted lines. Three different areas of each sample were analyzed; the representative results are shown. (d) Box plots; the rate of $\beta$-catenin-expressed cells. At least 300 cells were counted in randomly selected microscopic fields. The control values are indicated in blue, DCCs in red. The results shown are representative of at least three independent experiments with consistent results.

Dysfunction of the $\mathrm{Wnt} / \beta$-catenin signaling pathway may be a factor, resulting in follicle atresia, lower fertilization rate, or abnormal embryogenesis, compared to other cumulus cells. These results suggest that DCCs are indeed present in atretic follicles and can be distinguished from other cumulus cells in the maturation process by morphological evaluation.

\section{Discussion}

In this study, we found that DCCs showed the most variations in the activation of the Wnt signaling pathway compared to the control. It has been reported that the importance of the $\mathrm{Wnt} / \beta$-catenin signaling pathway in human cumulus cell function is related to follicle development and folliculogenesis [20-23]. DCCs were suggested to have different follicular development from that of control. The Wnt signaling pathway has two activation mechanisms, depending on $\beta$-catenin or not. Cytoplasmic $\beta$-catenin is kept at low levels through continuous proteasome-mediated degradation, which is controlled by the GSK-3/APC/AXIN complex. When ligands bind to receptors FZD and lipoprotein receptor-related proteins 5 and 6 (LRP5/6), the degradation of $\beta$-catenin is inhibited due to dissociation of $\beta$-catenin degradation complex, and consequently, $\beta$-catenin accumulates in the cytoplasm and nucleus. Nuclear $\beta$-catenin interacts with transcription factors, such as lymphoid enhancer-binding factor $1 / \mathrm{T}$ cell-specific transcription factor (LEF/TCF) to affect transcription [20,24]. 
Among the 19 Wnt ligands identified in humans, DCC showed a remarkable decrease in the expression of two ligands, $W N T 10 B$ and $W N T 9 B$. $W N T 10 B$ reportedly functions in the $W n t / \beta$-catenin signaling pathway [21]. While there are no reports directly associating $W N T 9 B$ with $W n t / \beta$-catenin signaling, WNT9B and WNT3 or WNT10B and WNT1 are closely located on the same chromosome of $17 \mathrm{q} 21$ or $12 \mathrm{q} 13$. WNT1, 2, 3, and $3 a$ [25] ligands have strong transformation abilities, leading to the activation of the $\mathrm{Wnt} / \beta$-catenin signaling pathway, and conceivably, it may be associated with $\beta$-catenin activation. Especially, WNT1 strongly transforms C57MC mammary epithelial cells [25] and participates in the activation of the Wnt/ $\beta$-catenin signaling pathway. Accordingly, WNT9B and WNT10B likely disturb the activation of the Wnt/ $\beta$-catenin signaling pathway. In immunostaining, localization of $\beta$-catenin in DCCs was different from that in the control, and the number of $\beta$-catenin-expressing cells in the nucleus was also significantly lower. In addition, in the control, the nuclei are round and regular, while the nuclei of DCC shows irregular form, likely nuclear fragmentation, implying that DCC may be a state of apoptosis during atresia. These results suggested that the $\mathrm{Wnt} / \beta$-catenin signaling pathway is inactivated in DCCs. Ten FZD members are capable of functioning as Wnt receptors [26]. There is no published report describing a direct relationship between FZD7 and cumulus cells. Conceivably, FZD7 expression is considered to be reduced, because the $\mathrm{Wnt} / \beta$-catenin signaling pathway is not active. When the Wnt $/ \beta$-catenin signaling pathway is inactive, AXIN forms a complex with APC and GSK-3 $\beta$ to act on $\beta$-catenin degradation [23]. DCCs also showed a decrease in AXIN expression, possibly due to inadequate transcription of $A X I N$, without the complex formation in the $\mathrm{Wnt} / \beta$-catenin signaling pathway. Furthermore, due to lower levels of intracellular $\beta$-catenin, the target genes, such as CCND1, could not be transcribed, resulting in a decreased expression.

Besides the genes related to Wnt signaling pathway and pluripotency, we found that six pathways were significantly downregulated (Table 1). Among them, G protein-coupled receptors (GPCRs) and Netrin-1 are reportedly associated with the Wnt signaling pathway. GPCRs comprise a very large family of proteins that play an essential role in cell proliferation, differentiation, neurotransmission, physiological processes, development, and apoptosis [27]. FZD family, the receptors for Wnt signaling, include GPCR [28], and the present results suggested that downregulation of FZD7 led to a decrease in GPCR activity. Netrin- 1 is the first axon guidance molecule found in vertebrates that has potent chemotaxis functions for axon guidance, cell migration, morphogenesis, and angiogenesis [29]. Netrin-1 has been reported to significantly promote $\beta$-catenin, which is vital for Wnt signaling after spinal cord injury [30], and reduced Netrin-1 activity in cumulus cells may negatively affect Wnt signaling activity. Moreover, regarding the other four pathways, the pancreatic cancer pathway is comprehensive, including the cell cycle and apoptosis. It is reportedly involved in the inhibition of pancreatic cancer via apoptosis [31] and may also be involved in cumulus cell apoptosis. Platelet homeostasis pathway reportedly plays an important role in the regulation of cell coagulation and hemostasis, as well as being involved in the regulation of angiogenesis and apoptosis [32]. Cumulus cells may be similarly affected by the decline in the platelet homeostasis pathway. Since arachidonic acid metabolism by cyclooxygenase (COX) is a major pathway for platelet activation [33], a decrease in arachidonic acid metabolic activity may have also reduced platelet homeostasis activity and led to apoptosis. In the integration of the energy metabolism, mitochondria play a central role, and they may be associated with apoptosis regulation [34,35]. Although these four pathways induce apoptosis as a result of their decreased activity, they are not associated with follicular development. Therefore, we did not consider them important and concentrated on the Wnt signaling pathway.

In conclusion, the DCC developed via controlled ovarian stimulation during the oocyte retrieval cycle suggests that dysfunction of the Wnt/ $\beta$-catenin signaling pathway may be a factor, resulting in follicle atresia, lower fertilization rate, or abnormal embryogenesis compared to other cumulus cells. These results suggest that DCCs are indeed present in atretic follicles and can be distinguished from other cumulus cells in the maturation process by morphological evaluation. Gene expression analysis of cumulus cells is a useful and safe method that evaluates the condition of oocytes. Based on the 
gene expression patterns of cumulus cells obtained in this study, there is evidence that they largely contribute to the development of future ART.

Supplementary Materials: The following are available online at http://www.mdpi.com/2673-3897/1/3/16/s1, Figure S1a: Three-dimensional scatter plots of gene expression using principal component analysis (PCA), Figure S1b: Venn diagrams of genes overlapped between DCCs and the control with two-fold differential expression, Table S1: Information on cumulus cells and patients age, Table S2: Gene type of UCSC transcript database and differential gene expression in DCCs, Table S3: Raw read count and mapped read count in DCCs and control, Table S4: Differentially expressed genes list in DCCs, Table S5: Gene Ontology analysis, Table S6: Upregulated pathways in DCCs.

Author Contributions: R.A., D.M., S.T., A.S., T.S., T.K. and H.S. conceived and designed the experiments. R.K.-M., M.A., K.T., E.I. and W.K. performed the experiments. R.K.-M., K.M. and T.K. analyzed the data. R.A., D.M., R.K.-M. and W.K. wrote the manuscript and prepared figures. All authors reviewed the manuscript. All authors have read and agreed to the published version of the manuscript.

Funding: This work was supported by the Ministry of Education, Culture, Sports, Science, and Technology Supported Program for the Strategic Research Foundation at Private Universities (S1311017).

Acknowledgments: We thank Keisuke Tanaka and Hiroto Sekitoh, affiliated to Tokyo University of Agriculture, for providing technical support.

Conflicts of Interest: The authors declare no conflict of interest. The funders had no role in the design of the study; in the collection, analyses, or interpretation of data; in the writing of the manuscript, or in the decision to publish the results.

\section{References}

1. Zhang, X.; Jafari, N.; Barnes, R.B.; Confino, E.; Milad, M.; Kazer, R.R. Studies of gene expression in human cumulus cells indicate pentraxin 3 as a possible marker for oocyte quality. Fertil. Steril. 2005, 83 (Suppl. 1), 1169-1179. [CrossRef] [PubMed]

2. Heller, D.T.; Schultz, R.M. Ribonucleoside metabolism by mouse oocytes: Metabolic cooperativity between the fully grown oocyte and cumulus cells. J. Exp. Zool. 1980, 214, 355-364. [CrossRef] [PubMed]

3. Brower, P.T.; Schultz, R.M. Intercellular communication between granulosa cells and mouse oocytes: Existence and possible nutritional role during oocyte growth. Dev. Biol. 1982, 90, 144-153. [CrossRef]

4. Larsen, W.J.; Wert, S.E.; Brunner, G.D. A dramatic loss of cumulus cell gap junctions is correlated with germinal vesicle breakdown in rat oocytes. Dev. Biol. 1986, 113, 517-521. [CrossRef]

5. Dekel, N.; Beers, W.H. Development of the rat oocyte in vitro: Inhibition and induction of maturation in the presence or absence of the cumulus oophorus. Dev. Biol. 1980, 75, 247-254. [CrossRef]

6. Lobo, R.A.; di Zerega, G.S.; Marrs, R.P. Follicular fluid steroid levels in dysmature and mature follicles from spontaneous and hyperstimulated cycles in normal and anovulatory women. J. Clin. Endocrinol. Metab. 1985, 60, 81-87. [CrossRef]

7. Tsuiki, A.; Preyer, J.; Hung, T.T. Fibronectin and glycosaminoglycans in human preovulatory follicular fluid and their correlation to follicular maturation. Hum. Reprod. 1988, 3, 425-429. [CrossRef]

8. Lefèvre, B.; Gougeon, A.; Nomé, F.; Testart, J. Effect of a gonadotropin-releasing hormone agonist and gonadotropins on ovarian follicles in cynomolgus monkey: A model for human ovarian hyperstimulation. Fertil. Steril. 1991, 56, 119-125. [CrossRef]

9. Morgan, P.M.; Boatman, D.E.; Bavister, B.D. Relationships between follicular fluid steroid hormone concentrations, oocyte maturity, in vitro fertilization and embryonic development in the rhesus monkey. Mol. Reprod. Dev. 1990, 27, 145-151. [CrossRef]

10. Ou, Y.C.; Lan, K.C.; Chang, S.Y.; Kung, F.T.; Huang, F.J. Increased progesterone/estradiol ratio on the day of HCG administration adversely affects success of in vitro fertilization-embryo transfer in patients stimulated with gonadotropin-releasing hormone agonist and recombinant follicle-stimulating hormone. Taiwan J. Obstet. Gynecol. 2008, 47, 168-174. [CrossRef]

11. Gougeon, A. Dynamics of follicular growth in the human: A model from preliminary results. Hum. Reprod. 1986, 1, 81-87. [CrossRef] [PubMed]

12. Kang, W.; Ishida, E.; Yamatoya, K.; Nakamura, A.; Miyado, M.; Miyamoto, Y.; Iwai, M.; Tatsumi, K.; Saito, T.; Saito, K.; et al. Autophagy-disrupted LC3 abundance leads to death of supporting cells of human oocytes. Biochem. Biophys. Rep. 2018, 15, 107-114. [CrossRef] [PubMed] 
13. Ito, M.; Muraki, M.; Takahashi, Y.; Imai, M.; Tsukui, T.; Yamakawa, N.; Nakagawa, K.; Ohgi, S.; Horikawa, T.; Iwasaki, W.; et al. Glutathione S-transferase theta 1 expressed in granulosa cells as a biomarker for oocyte quality in age-related infertility. Fertil. Steril. 2008, 90, 1026-1035. [CrossRef] [PubMed]

14. Hu, M.-H.; Zheng, S.-X.; Yin, H.; Zhu, X.-Y.; Lu, F.-T.; Tong, X.-H.; Liu, Y.-S.; Zhang, Y.-W.; Xu, B. Identification of microRNAs that Regulate the MAPK Pathway in Human Cumulus Cells from PCOS Women with Insulin Resistance. Reprod. Sci. 2020, 27, 833-844. [CrossRef]

15. Xu, Y.; Zhou, T.; Shao, L.; Zhang, B.; Liu, K.; Gao, C.; Gao, L.; Liu, J.; Cui, Y.; Chian, R.-C.; et al. Gene expression profiles in mouse cumulus cells derived from in vitro matured oocytes with and without blastocyst formation. Gene Expr. Patterns 2017, 25-26, 46-58. [CrossRef]

16. Weizman, N.F.; Wyse, B.A.; Gat, I.; Balakier, H.; Sangaralingam, M.; Caballero, J.; Kenigsberg, S.; Librach, C.L. Triggering method in assisted reproduction alters the cumulus cell transcriptome. Reprod. Biomed. Online 2019, 39, 211-224. [CrossRef]

17. Xu, B.; Zhang, Y.W.; Tong, X.H.; Liu, Y.S. Characterization of microRNA profile in human cumulus granulosa cells: Identification of microRNAs that regulate Notch signaling and are associated with PCOS. Mol. Cell Endocrinol. 2015, 404, 26-36. [CrossRef] [PubMed]

18. Chronowska, E. High-throughput analysis of ovarian granulosa cell transcriptome. BioMed Res. Int. 2014, 2014, 213570. [CrossRef]

19. Mortazavi, A.; Williams, B.A.; McCue, K.; Schaeffer, L.; Wold, B. Mapping and quantifying mammalian transcriptomes by RNA-Seq. Nat. Methods 2008, 5, 621-628. [CrossRef]

20. Wang, H.X.; Tekpetey, F.R.; Kidder, G.M. Identification of WNT/beta-CATENIN signaling pathway components in human cumulus cells. Mol. Hum. Reprod. 2009, 15, 11-17. [CrossRef]

21. Harwood, B.N.; Cross, S.K.; Radford, E.E.; Haac, B.E.; De Vries, W.N. Members of the WNT signaling pathways are widely expressed in mouse ovaries, oocytes, and cleavage stage embryos. Dev. Dyn. 2008, 237, 1099-1111. [CrossRef] [PubMed]

22. Hsieh, M.; Johnson, M.A.; Greenberg, N.M.; Richards, J.S. Regulated expression of Wnts and Frizzleds at specific stages of follicular development in the rodent ovary. Endocrinology 2002, 143, 898-908. [CrossRef] [PubMed]

23. Hernandez Gifford, J.A. The role of WNT signaling in adult ovarian folliculogenesis. Reproduction 2015, 150, R137-R148. [CrossRef]

24. Logan, C.Y.; Nusse, R. The Wnt signaling pathway in development and disease. Annu. Rev. Cell Dev. Biol. 2004, 20, 781-810. [CrossRef] [PubMed]

25. Shimizu, H.; Julius, M.A.; Giarre, M.; Zheng, Z.; Brown, A.M.; Kitajewski, J. Transformation by Wnt family proteins correlates with regulation of beta-catenin. Cell Growth Differ. 1997, 8, 1349-1358.

26. Bjarnadottir, T.K.; Gloriam, D.E.; Hellstrand, S.H.; Kristiansson, H.; Fredriksson, R.; Schioth, H.B. Comprehensive repertoire and phylogenetic analysis of the $\mathrm{G}$ protein-coupled receptors in human and mouse. Genomics 2006, 88, 263-273. [CrossRef]

27. Nieto Gutierrez, A.; McDonald, P.H. GPCRs: Emerging anti-cancer drug targets. Cell Signal. 2018, 41, 65-74. [CrossRef]

28. Lynch, J.R.; Wang, J.Y. G Protein-Coupled Receptor Signaling in Stem Cells and Cancer. Int. J. Mol. Sci. 2016, 17, 707. [CrossRef]

29. Tepavčević, V.; Bs, C.K.; Bs, M.S.A.; Meppiel, E.; Ms, S.M.; Ms, R.A.; Ravassard, P.; Kennedy, T.E.; Nait-Oumesmar, B.; Lubetzki, C. Early netrin-1 expression impairs central nervous system remyelination. Ann. Neurol. 2014, 76, 252-268. [CrossRef]

30. Gao, K.; Niu, J.; Dang, X. Neuroprotection of netrin-1 on neurological recovery via Wnt/ $\beta$-catenin signaling pathway after spinal cord injury. Neuroreport 2020, 31, 537-543. [CrossRef]

31. Li, Y.; Li, L.; Zhang, G.; Wang, Y.; Chen, H.; Kong, R.; Pan, S.; Sun, B. Crucial microRNAs and genes in metformin's anti-pancreatic cancer effect explored by microRNA-mRNA integrated analysis. Investig. New Drugs 2018, 36, 20-27. [CrossRef] [PubMed]

32. Eisinger, F.; Patzelt, J.; Langer, H.F. The Platelet Response to Tissue Injury. Front. Med. 2018, 5, 317. [CrossRef] [PubMed]

33. Bijak, M.; Saluk-Bijak, J. Flavonolignans inhibit the arachidonic acid pathway in blood platelets. BMC Complement. Altern. Med. 2017, 17, 396. [CrossRef] [PubMed] 
34. Vakifahmetoglu-Norberg, H.; Ouchida, A.T.; Norberg, E. The role of mitochondria in metabolism and cell death. Biochem. Biophys. Res. Commun. 2017, 482, 426-431. [CrossRef]

35. Susin, S.A.; Lorenzo, H.K.; Zamzami, N.; Marzo, I.; Snow, B.E.; Brothers, G.M.; Mangion, J.; Jacotot, E.; Costantini, P.; Loeffler, M.; et al. Molecular characterization of mitochondrial apoptosis-inducing factor. Nature 1999, 397, 441-446. [CrossRef]

Publisher's Note: MDPI stays neutral with regard to jurisdictional claims in published maps and institutional affiliations.

(C) 2020 by the authors. Licensee MDPI, Basel, Switzerland. This article is an open access article distributed under the terms and conditions of the Creative Commons Attribution (CC BY) license (http://creativecommons.org/licenses/by/4.0/). 